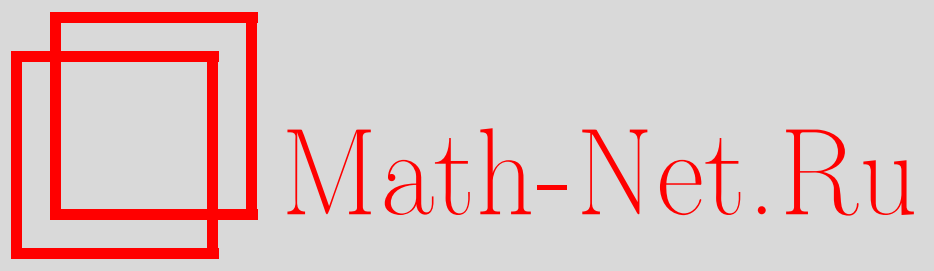

В. Б. Бобров, Диэлектрическая проницаемость и одночастичные функции распределения в термодинамике кулоновской системы, ТМФ, 2014, том 179, номер 2, 251-266

DOI: https://doi.org/10.4213/tmf8640

Использование Общероссийского математического портала Math-Net.Ru подразумевает, что вы прочитали и согласны с пользовательским соглашением http://www.mathnet.ru/rus/agreement

Параметры загрузки:

IP: 54.157 .27 .8

26 апреля 2023 г., 12:44:23

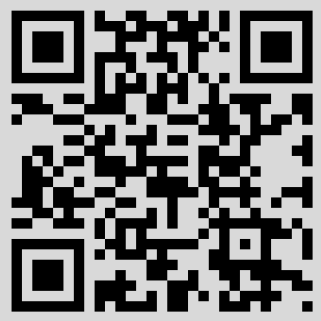




\title{
ДИЭЛЕКТРИЧЕСКАЯ ПРОНИЦАЕМОСТЬ И ОДНОЧАСТИЧНЫЕ ФУНКЦИИ РАСПРЕДЕЛЕНИЯ В ТЕРМОДИНАМИКЕ КУЛОНОВСКОЙ СИСТЕМЫ
}

\begin{abstract}
С использованием большого канонического распределения и теоремы вириала показано, что термодинамический потенциал Гиббса нерелятивистской системы заряженных частиц однозначно определяется ее диэлектрической проницаемостью и функциями распределения электронов и ядер вне рамок теории возмущений. Это означает, что для вычисления термодинамических функций кулоновской системы следует использовать согласованные приближения для диэлектрической проницаемости и одночастичных функций распределения электронов и ядер. Для построения таких самосогласованных приближений предлагается применять в уравнениях движения процедуру расцепления, основанную на выделении "связных" и "регулярных" частей температурных функций Грина. Рассмотрено самосогласованное приближение Хартри-Фока, отвечающее этой процедуре.
\end{abstract}

Ключевые слова: кулоновская модель вещества, диэлектрическая проницаемость, теорема вириала, одночастичная функция распределения, температурные функции Грина.

DOI: $10.4231 / \operatorname{tmf} 8640$

\section{1. ВВЕДЕНИЕ}

В широкой области термодинамических параметров свойства вещества наиболее адекватно описываются в модели нерелятивистской системы заряженных частиц (электронов и ядер), взаимодействующих между собой посредством кулоновского потенциала (кулоновская система, КС) [1]. Сложности в теоретическом исследовании свойств КС обусловлены необходимостью одновременного учета коллективных эффектов, прежде всего экранирования кулоновского взаимодействия, и квантового описания, как вследствие образования связанных состояний электронов и ядер, так и вследствие необходимости учитывать тождественность элементарных частиц [2].

* Объединенный институт высоких температур РАН, Москва, Россия. E-mail: vic5907@mail.ru

†Национальный исследовательский университет "Московский энергетический институт", Москва, Россия 
Принципиальная возможность разрешения возникающих проблем основана на использовании различных вариантов диаграммной техники теории возмущений по межчастичному взаимодействию, разработанных в рамках методов квантовой теории поля (см., например, монографии [3], [4]). При рассмотрении термодинамических свойств $\mathrm{KC}$, находящейся в объеме $V$ при температуре $T$ (в энергетических единицах), задача сводится к вычислению термодинамического потенциала $\Omega\left(V, T,\left\{\mu_{a}\right\}\right)$ :

$$
\Omega=-T \ln \left(\operatorname{Tr} \exp \left\{-\frac{\widehat{H}-\sum_{a} \mu_{a} \widehat{N}_{a}}{T}\right\}\right),
$$

где гамильтониан $\mathrm{KC} \mathrm{равен}$

$$
\begin{gathered}
\widehat{H}=\widehat{H}^{(0)}+\widehat{U}^{\mathrm{int}}, \quad \widehat{H}^{(0)}=\sum_{a} \sum_{\vec{p}, \sigma} \epsilon_{a}(p) \hat{a}_{\vec{p}, \sigma}^{+} \hat{a}_{\vec{p}, \sigma}, \quad \widehat{U}^{\mathrm{int}}=\sum_{a, b} \widehat{U}_{a b}, \\
\widehat{U}_{a b}=\frac{1}{2 V} \sum_{\vec{q} \neq 0} \sum_{\vec{p}, \sigma} u_{a b}(q) \hat{a}_{\vec{p}_{1}+\vec{q} / 2, \sigma_{1}}^{+} \hat{b}_{\vec{p} 2}^{+}-\vec{q} / 2, \sigma_{2} \\
\hat{b}_{\vec{p}_{2}+\vec{q} / 2, \sigma_{2}} \hat{a}_{\vec{p}_{1}-\vec{q} / 2, \sigma_{1}} .
\end{gathered}
$$

Здесь $\hat{a}_{\vec{p}, \sigma}^{+}$и $\hat{a}_{\vec{p}, \sigma}-$ соответственно операторы рождения и уничтожения в состоянии с импульсом $\hbar \vec{p}$ и спиновым числом $\sigma$ для частиц сорта $a$, которые характеризуются массой $m_{a}$, зарядом $z_{a} e$ и химическим потенциалом $\mu_{a} ; \widehat{N}_{a}=\sum_{\vec{p}, \sigma} \hat{a}_{\vec{p}, \sigma}^{+} \hat{a}_{\vec{p}, \sigma}$ - оператор полного числа частиц сорта $a ; \epsilon_{a}(p)=\hbar^{2} p^{2} / 2 m_{a}$ - энергия свободной частицы сорта $a ; u_{a b}(q)=4 \pi z_{a} z_{b} e^{2} / q^{2}$ - фурье-компонента потенциала кулоновского взаимодействия частиц сортов $a$ и $b$. При этом химические потенциалы $\mu_{a}$ различных сортов частиц рассматриваются как формально независимые величины при учете условия квазинейтральности

$$
\sum_{a} z_{a} e n_{a}=0
$$

на конечном этапе вычислений физических величин (см. подробнее работу [5]). Здесь

$$
n_{a}\left(T,\left\{\mu_{b}\right\}\right)=\frac{\left\langle\widehat{N}_{a}\right\rangle}{V}=-\left(\frac{\partial \Omega}{\partial \mu_{a}}\right)_{V, T, \mu_{b} \neq \mu_{a}}
$$

- средняя плотность числа частиц сорта $a$ в объеме $V$, угловые скобки $\langle\cdot\rangle$ обозначают усреднение с большим каноническим распределением.

Для вычисления термодинамического потенциала Гиббса $\Omega\left(V, T,\left\{\mu_{a}\right\}\right)$ в теории равновесных $\mathrm{KC}$ используются три основных подхода. Один из этих подходов основан на использовании равенства [6]

$$
\Omega-\Omega^{(0)}=-T \ln \langle\widehat{S}(\beta)\rangle^{(0)}, \quad \widehat{S}(\beta)=\widehat{T}_{\tau} \exp \left\{-\int_{0}^{\beta} d \tau \widehat{U}^{\mathrm{int}}(\tau)\right\},
$$

где индекс (0) обозначает, что соответствующая величина отвечает системе невзаимодействующих частиц с гамильтонианом $H^{(0)}$, заданным в $(2), \widehat{S}(\beta)$ - матрица Мацубары,

$$
\langle\widehat{S}(\beta)\rangle^{(0)}=\sum_{n} \frac{(-1)^{n}}{n !} \int_{0}^{\beta} \ldots \int_{0}^{\beta} d \tau_{1} \ldots d \tau_{n}\left\langle\widehat{T}_{\tau}\left\{\widehat{U}^{\mathrm{int}}\left(\tau_{1}\right) \ldots \widehat{U}^{\mathrm{int}}\left(\tau_{n}\right)\right\}\right\rangle^{(0)},
$$


$\widehat{T}_{\tau}$ - оператор $\tau$-упорядочения, $\beta=1 / T$ - обратная температура,

$$
\widehat{U}^{\mathrm{int}}(\tau)=\exp \left\{\left(\widehat{H}^{(0)}-\sum_{a} \mu_{a} \widehat{N}_{a}\right) \tau\right\} \widehat{U}^{\mathrm{int}} \exp \left\{-\left(\widehat{H}^{(0)}-\sum_{a} \mu_{a} \widehat{N}_{a}\right) \tau\right\} .
$$

Как видно из соотношения (5), величина $\langle\widehat{S}(\beta)\rangle^{(0)}$ представлена в виде ряда по параметру взаимодействия $\lambda=e^{2}$, поэтому в рамках теории возмущений можно было бы решить задачу о вычислении термодинамического потенциала с использованием одночастичных температурных функций Грина невзаимодействующих частиц [4]. Однако для $\mathrm{KC}$ такой подход не может быть реализован непосредственно из-за наличия расходимостей в отдельных членах ряда теории возмущений в силу дальнодействующего характера кулоновского взаимодействия. По-видимому, впервые решение этой проблемы с помощью процедуры разложения в ряд теории возмущений по так называемому экранированному потенциалу кулоновского взаимодействия $u_{a b}^{\mathrm{S}}\left(q, \omega_{l}\right)$, зависящему от мнимых частот $i \omega_{l}\left(\omega_{l}=2 \pi l, l=0, \pm 1, \ldots\right)$, вместо разложения по "голому" потенциалу $u_{a b}(q)$ было рассмотрено в статье [7]. Подробное описание применения этого подхода для определения термодинамических величин газовой плазмы водорода представлено в работе [8].

Однако при вычислении величины $\langle\widehat{S}(\beta)\rangle^{(0)}$ фактически отсутствуют правила построения согласованных приближений, связанных с разложением по экранированному потенциалу $u_{a b}^{\mathrm{S}}\left(q, \omega_{l}\right)$. Это обстоятельство не позволяет дать четкое обоснование используемых приближений при вычислении $\langle\widehat{S}(\beta)\rangle^{(0)}$. В связи с этим при вычислении термодинамического потенциала $\Omega\left(V, T,\left\{\mu_{a}\right\}\right)$, заданного в $(1)$, для КС вместо соотношения (5) часто используется равенство [2]

$$
\Omega-\Omega^{(0)}=\int_{0}^{1} d \lambda\left\langle\widehat{U}^{\mathrm{int}}\right\rangle_{\lambda}
$$

где индекс $\lambda$ обозначает, что усреднение по большому каноническому распределению производится с гамильтонианом $\widehat{H}_{\lambda}=\widehat{H}^{(0)}+\lambda \widehat{U}^{\text {int }}$. При реализации этого подхода обычно используется диаграммная техника Каданова-Бейма [3], основанная на рассмотрении временных функций Грина и позволяющая единообразно анализировать как равновесные, так и неравновесные свойства КС (см, например, книгу [9]). Величина $\left\langle\widehat{U}^{\text {int }}\right\rangle_{\lambda}$ полностью определяется двухчастичной функцией Грина, для вычисления которой существуют определенные разложения по экранированному потенциалу взаимодействия $u_{a b}^{\mathrm{S}}$, детальное обсуждение этих разложений представлено в монографии [2]. Однако при использовании равенства (6) возникает проблема самосогласования, связанная с интегрированием по параметру взаимодействия $\lambda$ и обусловленная приближенным вычислением величины $\left\langle\widehat{U}^{\text {int }}\right\rangle_{\lambda}$. Эта проблема хорошо известна в теории вырожденной электронной жидкости [10]. Современное состояние подхода, основанного на диаграммной технике Каданова-Бейма, представлено в книгах [9], [11].

Альтернативный подход к рассмотрению термодинамических свойств КС был предложен Монтроллом и Уордом, которые обобщили метод групповых интегралов Майера и соответствующую диаграммную технику на квантовый случай [12]. Каноническая статистическая сумма в этом подходе явно выражается через функцию 
распространения, которая удовлетворяет уравнению Блоха, а для системы невзаимодействующих частиц записывается через произведение одночастичных функций распространения с учетом тождественности частиц. Способ устранения расходимостей отдельных групповых интегралов для полностью ионизованной плазмы на основе суммирования так называемых кольцевых диаграмм был рассмотрен в работе [13]. Роджерс и Девитт дали систематическое изложение этого подхода с возможностью учета связанных состояний на основе экранированного кластерного разложения [14]. Дальнейшее развитие этого метода дано в работе [15]. В отличие от двух других подходов, основанных на формализме функций Грина, правила вычисления диаграмм в рамках экранированного кластерного разложения весьма сложны. Это обстоятельство ограничивает возможности аналитического исследования с помощью простейших приближений. Однако существенным преимуществом этого подхода является возможность непосредственного применения метода Монте-Карло расчета интегралов по траекториям для вычисления термодинамических свойств КС вне рамок теории возмущений (см. работы [15]-[17] и цитированную в них литературу). Таким образом, подход Монтролла-Уорда может быть использован для исследования равновесных свойств КС численными методами, а также для сравнения полученных таким образом результатов с аналитическими результатами применения двух других подходов, основанных на формализме функций Грина. При этом мы исходим из того, что все три подхода, изложенные выше, при точном рассмотрении эквивалентны.

Однако конкретные аналитические вычисления при использовании этих методов ограничиваются учетом некоторого ограниченного числа членов разложений в рядах теории возмущений. При этом эквивалентные результаты получаются, только если рассматривать простейшие приближения, отвечающие низшим порядкам теории возмущений. В частности, до настоящего времени известная проблема вычисления конечной статистической суммы "атома" водорода в разреженной плазме не получила однозначного решения (см. статьи [18]-[20] и цитированную в них литературу).

На наш взгляд, имеющиеся расхождения обусловлены отсутствием в каждом из рассмотренных подходов правил построения самосогласованных приближений, основанных на использовании ограниченного числа диаграмм. При формулировке таких правил необходимо учитывать точные соотношения (или правила сумм), которые должны выполняться вне рамок теории возмущений как для вычисляемых термодинамических функций, так и для функций Грина.

В качестве иллюстрации рассмотрим следующий пример. Как уже было отмечено выше, разложение в ряд теории возмущений вследствие специфики кулоновского взаимодействия должно осуществляться по экранированному потенциалу взаимодействия, который равен

$$
u_{a b}^{\mathrm{S}}\left(q, \omega_{l}\right)=\frac{u_{a b}(q)}{\varepsilon\left(q, i \omega_{l}\right)},
$$

где $\varepsilon\left(q, i \omega_{l}\right)=1-u(q) \Pi\left(q, i \omega_{l}\right)$ - диэлектрическая проницаемость (ДП) КС; здесь $u(q)=4 \pi / q^{2}, \Pi\left(q, i \omega_{l}\right)$ - так называемый поляризационный оператор “заряд-заряд”, который является неприводимой частью функции отклика "заряд-заряд" $\chi_{z z}\left(q, i \omega_{l}\right)$ по одной линии кулоновского взаимодействия (см., например, монографии [2], [3]). 
При этом для статического значения поляризационного оператора П $(q, 0)$ в длинноволновом пределе $q \rightarrow 0$ имеет место точное равенство (см. работы [21], [22] и цитированную в них литературу)

$$
\lim _{q \rightarrow 0} \Pi(q, 0)=-\sum_{a, b} z_{a} z_{b} e^{2}\left(\frac{\partial n_{a}}{\partial \mu_{b}}\right)_{T} .
$$

Учтем теперь, что для вычисления средней плотности $n_{a}$ мы можем использовать равенство [4]

$$
n_{a}\left(T,\left\{\mu_{b}\right\}\right)=\sum_{\sigma} \int \frac{d^{3} p}{(2 \pi)^{3}} f_{a}(p, \sigma),
$$

где $f_{a}(p, \sigma)=\left\langle a_{\vec{p}, \sigma}^{+} a_{\vec{p}, \sigma}\right\rangle$ - одночастичная функция распределения (ОЧФР) (среднее число заполнения) для частиц сорта $a$, которая зависит также от температуры $T$ и различных химических потенциалов $\left\{\mu_{b}\right\}$. Для системы невзаимодействующих частиц функция $f_{a}(p, \sigma)$ определяется распределениями Ферми-Дирака или Бозе-Эйнштейна. В свою очередь,

$$
f_{a}(p, \sigma)= \pm \lim _{\tau \rightarrow+0} \sum_{\omega_{n}} g_{a}\left(p, \sigma ; \omega_{n}\right) e^{i \omega_{n} \tau}
$$

Здесь $g_{a}\left(p, \sigma ; \omega_{n}\right)$ - точная температурная одночастичная функция Грина (ОЧФГ) для частиц сорта $a$ в технике Мацубары, знак плюс в соотношении (10) отвечает статистике Бозе-Эйнштейна (в этом случае $\omega_{n}=i 2 \pi n$ ), знак минус отвечает статистике Ферми-Дирака (в этом случае $\omega_{n}=i \pi(2 n+1)$, где $n$ - целое число). В технике Каданова-Бейма, где ОЧФГ зависит от частоты как от непрерывной переменной и полностью определяется одночастичной корреляционной функцией $g_{a}^{<}(p, \sigma ; \omega)$ (см. подробнее монографии [2], [3]), равенство для ОЧФР, соответствующее (10), имеет вид

$$
f_{a}(p, \sigma)=\int_{-\infty}^{+\infty} \frac{d \omega}{2 \pi} g_{a}^{<}(p, \sigma ; \omega) .
$$

Таким образом, с учетом соотношений (7)-(11) мы приходим к выводу, что при последовательном рассмотрении термодинамических свойств КС используемое приближение для ОЧФГ должно быть согласовано с соответствующим приближением для экранированного потенциала $u_{a b}^{\mathrm{S}}$. Необходимость такого согласования является следствием того, что ДП для КС представляет собой функционал от ОЧФГ электронов и ядер. Это утверждение непосредственно следует из диаграммного разложения для ДП. В частности, широко используемое в теории плазмы приближение хаотических фаз для ДП соответствует ОЧФГ для невзаимодействующих частиц [2]. Иными словами, при учете эффектов взаимодействия в ОЧФГ необходимо учитывать аналогичные эффекты взаимодействия в ДП, и наоборот. Но процедура такого согласования не рассматривается ни в одном из изложенных выше подходов при вычислении термодинамических свойств $\mathrm{KC}$, что и является, на наш взгляд, причиной расхождений в получаемых результатах. В большинстве случаев даже при рассмотрении сильно неидеальной КС для ДП используется приближение хаотических фаз, которое обеспечивает устранение кулоновской расходимости. 


\section{2. ТЕРМОДИНАМИЧЕСКИЙ ПОТЕНЦИАЛ ДЛЯ КС И ТЕОРЕМА ВИРИАЛА}

Мы можем убедиться в необходимости согласования используемых приближений для ОЧФГ и ДП и без привлечения методов теории возмущений. Применим для этого теорему вириала, которая для $\mathrm{KC}$ имеет вид

$$
P V=\frac{2}{3}\left\langle\widehat{H}^{(0)}\right\rangle+\frac{1}{3}\left\langle\widehat{U}^{\text {int }}\right\rangle .
$$

Обратим внимание, что теорема вириала справедлива при использовании любого из распределений Гиббса для описания равновесных систем (см., например, монографию [23]), вследствие того что теорему вириала можно получить непосредственно из стационарного уравнения Шредингера [24], [25]. Это означает, что в соотношении (12) мы можем рассматривать давление как функцию $P\left(T,\left\{\mu_{b}\right\}\right)$ или, что эквивалентно, $P\left(T,\left\{\mu_{b}\right\}\right) V=-\Omega\left(V, T,\left\{\mu_{a}\right\}\right)$. Таким образом, теорему вириала можно использовать как альтернативный способ вычисления термодинамического потенциала Гиббса, а тем самым и любых термодинамических свойств КС.

В термодинамическом пределе $V \rightarrow \infty$ равенство (12) можно представить в виде

$$
\begin{aligned}
P= & \frac{2}{3} \sum_{a} \sum_{\sigma} \int \frac{d^{3} p}{(2 \pi)^{3}} \epsilon_{a}(p) f_{a}(p, \sigma)+ \\
& +\frac{1}{6} \sum_{a, b}\left(n_{a} n_{b}\right)^{1 / 2} \int \frac{d^{3} q}{(2 \pi)^{3}} u_{a b}(q)\left(S_{a b}(q)-\delta_{a, b}\right),
\end{aligned}
$$

где $S_{a b}(q)$ - так называемый статический структурный фактор для частиц сортов $a$ и $b$,

$$
\left(n_{a} n_{b}\right)^{1 / 2} S_{a b}(q)=\lim _{V \rightarrow \infty} \frac{1}{V}\left\langle\hat{\rho}_{\vec{q}}^{a} \hat{\rho}_{-\vec{q}}^{b}\right\rangle
$$

Здесь

$$
\hat{\rho}_{\vec{q}}^{a}=\sum_{\vec{p}} \sum_{\sigma} \hat{a}_{\vec{p}-\vec{q} / 2, \sigma}^{+} \hat{a}_{\vec{p}+\vec{q} / 2, \sigma}
$$

- фурье-компонента оператора плотности числа частиц сорта $a$.

Учтем определение потенциала кулоновского взаимодействия $u_{a b}(q)=4 \pi z_{a} z_{b} e^{2} / q^{2}$ и введем в рассмотрение статический структурный фактор “заряд-заряд"

$$
S_{z z}(q)=\sum_{a, b}\left(n_{a} n_{b}\right)^{1 / 2} z_{a} z_{b} e^{2} S_{a b}(q)
$$

Тогда соотношение (13) принимает вид

$$
P\left(T,\left\{\mu_{b}\right\}\right)=\frac{2}{3} \sum_{a} \sum_{\sigma} \int \frac{d^{3} p}{(2 \pi)^{3}} \epsilon_{a}(p) f_{a}(p, \sigma)+\frac{T}{6} \int \frac{d^{3} q}{(2 \pi)^{3}}\left(\frac{4 \pi S_{z z}(q)}{q^{2} T}-\frac{1}{q^{2} R_{\mathrm{D}}^{2}}\right),
$$


где $R_{\mathrm{D}}=\left(4 \pi \sum_{a} z_{a}^{2} e^{2} n_{a} / T\right)^{-1 / 2}-$ радиус Дебая. Из спектрального представления для (13), (14) непосредственно следует соотношение (см. подробности в [2], [3])

$$
S_{z z}(q)=-\frac{\hbar}{\pi} \int_{-\infty}^{+\infty} d \omega \frac{\operatorname{Im} \chi_{z z}^{\mathrm{R}}(q, \omega)}{1-e^{-\hbar \omega / T}}
$$

где $\chi_{z z}^{\mathrm{R}}(q, \omega)$ - так называемая запаздывающая функция отклика “заряд-заряд”,

$$
\chi_{z z}^{\mathrm{R}}(q, \omega)=-\frac{i}{\hbar V} \int_{0}^{\infty} d t e^{i \omega t}\left\langle\left[\hat{\rho}_{\vec{q}}^{z}(t), \hat{\rho}_{-\vec{q}}^{z}(0)\right]_{-}\right\rangle,
$$

и $\hat{\rho}_{\vec{q}}^{z}(t)=\sum_{a} z_{a} e \hat{\rho}_{\vec{q}}^{a}(t)$ - фурье-компонента оператора плотности заряда в представлении Гейзенберга. Соотношение (16) следует понимать в термодинамическом пределе $V \rightarrow \infty$.

В свою очередь, функция отклика $\chi_{z z}^{\mathrm{R}}(q, \omega)$ непосредственным образом определяет ДП $\varepsilon(q, \omega)$ для КС как ее электромагнитную характеристику в слабом скалярном электрическом поле в рамках теории линейного отклика (см. подробнее в книге [2]):

$$
\varepsilon^{-1}(q, \omega)=1+\frac{4 \pi}{q^{2}} \chi_{z z}^{\mathrm{R}}(q, \omega)
$$

Запаздывающие функции отклика (функции Грина) являются аналитическими функциями в верхней полуплоскости комплексных $\omega$ (для $\operatorname{Im} \omega>0$ ). При частотах $\hbar \omega=i 2 \pi l(l>0)$ на мнимой оси их значения совпадают со значениями соответствующих температурных функций отклика (см. подробнее в монографии [4]):

$$
\left.\chi_{z z}^{\mathrm{R}}(q, \hbar \omega)\right|_{\hbar \omega=i \omega_{l}}=\chi_{z z}\left(q, i \omega_{l}\right) .
$$

Иными словами, ДП в определении (7) экранированного потенциала $u_{a b}^{\mathrm{S}}$ является линейным электромагнитным свойством КС. При этом соотношение (16) с учетом формул (18), (19) можно записать в виде [21], [26]

$$
S_{z z}(q)=-T \sum_{\omega_{l}} \chi_{z z}\left(q, i \omega_{l}\right)=-\frac{q^{2} T}{4 \pi} \sum_{\omega_{l}}\left(\varepsilon^{-1}\left(q, i \omega_{l}\right)-1\right) .
$$

Таким образом, из соотношений (15)-(20) следует, что термодинамические свойства $\mathrm{KC} \mathrm{однозначно} \mathrm{определяются} \mathrm{ее} \mathrm{ДП} \mathrm{и} \mathrm{ОЧФР} \mathrm{электронов} \mathrm{и} \mathrm{ядер} \mathrm{вне} \mathrm{рамок}$ теории возмущений по межчастичному взаимодействию.

Учтем далее, что непосредственное вычисление ОЧФР электронов и ядер основано на использовании соответствующих ОЧФГ. При этом выполняется равенство, которое в технике Каданова-Бейма [3] имеет вид

$$
\left\langle U^{\text {int }}\right\rangle=\frac{1}{2} V \sum_{a} \sum_{\sigma} \int \frac{d^{3} p}{(2 \pi)^{3}} \int_{-\infty}^{+\infty} \frac{d \omega}{2 \pi}\left(\omega-\epsilon_{a}(p)\right) g_{a}^{<}(p, \sigma ; \omega) .
$$

Но согласно равенству (11) корреляционная функция $g_{a}^{<}(p, \sigma ; \omega)$ однозначно определяет соответствующую функцию распределения $f_{a}(p, \sigma)$. При этом средняя потенциальная энергия $\left\langle\widehat{U}^{\text {int }}\right\rangle$ в соответствии с формулами (15)-(20) однозначно определяется величиной ДП. 
В результате мы подтверждаем вывод о необходимости самосогласованного вычисления ОЧФР электронов и ядер и ДП для определения термодинамических свойств КС. Более того, из соотношений (2), (21) и теоремы вириала (12) непосредственно следует, что термодинамический потенциал $\Omega\left(V, T,\left\{\mu_{a}\right\}\right)$ или давление $P\left(T,\left\{\mu_{b}\right\}\right)$ для $\mathrm{KC} \mathrm{однозначно} \mathrm{определяются} \mathrm{точными} \mathrm{ОЧФГ} \mathrm{электронов} \mathrm{и} \mathrm{ядер} \mathrm{вне}$ рамок теории возмущений [27]:

$$
P\left(T,\left\{\mu_{b}\right\}\right)=\frac{1}{6} \sum_{a} \sum_{\sigma} \int \frac{d^{3} p}{(2 \pi)^{3}} \int_{-\infty}^{+\infty} \frac{d \omega}{2 \pi}\left(\omega+3 \epsilon_{a}(p)\right) g_{a}^{<}(p, \sigma ; \omega) .
$$

Данное утверждение находится в согласии с известным результатом Латтинджера и Уорда [28] о том, что термодинамический потенциал Гиббса для однокомпонентной системы является функционалом от ОЧФГ. Явный вид такого функционала может быть установлен только в рамках теории возмущений, хотя предпринимаются попытки решить эту проблему в рамках термодинамически самосогласованных приближений (см. работы [29], [30] и ссылки в них). Вывод явного выражения (22) для термодинамического потенциала Гиббса КС через точные ОЧФГ электронов и ядер опирается на то, что потенциал кулоновского взаимодействия является однородной функцией расстояния между заряженными частицами.

\section{3. УРАВНЕНИЯ ДВИЖЕНИЯ ДЛЯ ТЕМПЕРАТУРНЫХ ФУНКЦИЙ ГРИНА}

Таким образом, проблема вычисления термодинамических свойств КС сводится к определению ДП и ОЧФР электронов и ядер. При этом согласно проведенному выше рассмотрению основной проблемой является развитие методов самосогласованного вычисления ДП и ОЧФР электронов и ядер. Чтобы решить эту проблему, мы предлагаем использовать цепочку уравнений Боголюбова для двухвременных (запаздывающих или опережающих) функций Грина. В традиционной формулировке этот метод наталкивается на характерные трудности, связанные с необходимостью оборвать ("расцепить") получающуюся систему уравнений (см. подробности в работах [31], [32]). В то же время после решения приближенной замкнутой системы уравнений определение макроскопических характеристик осуществляется стандартными способами [33].

Возможный вариант общего построения бесконечной цепочки замкнутых уравнений для временных функций Грина был предложен в работе [34] на основе представления для "высших" функций Грина через так называемые "регулярные" части функций Грина и соответствующих средних более низкого порядка. В этом случае имеется возможность получить систему зацепляющихся интегральных уравнений относительно "регулярных" частей временных функций Грина. Проблема обоснования такого метода, связанная с невозможностью использования методов квантовой теории поля для запаздывающих или опережающих временных функций Грина, решена в работе [35], где соответствующая процедура была реализована для температурных функций Грина. Более того, в работе [36] показана возможность применения этого подхода для непосредственного вычисления ДП.

Суть предлагаемого метода получения самосогласованных уравнений сводится к следующему. Для определенности будем рассматривать заряженные частицы как 
фермионы со спином $1 / 2$. Из определения температурной ОЧФГ $g_{a}\left(p, \sigma ; \omega_{n}\right)$ непосредственно следует уравнение движения

$$
\begin{aligned}
\left(i \omega_{n}\right. & \left.-\epsilon_{a}(p)+\mu_{a}\right) g_{a}\left(p, \sigma ; \omega_{n}\right)= \\
& =1+V^{-1} \sum_{\vec{q} \neq 0} \sum_{\vec{p}_{1}, \sigma_{1}} u_{a b}(q)\left\langle\left\langle\hat{b}_{\vec{p}_{1}+\vec{q}, \sigma_{1}}^{+} \hat{b}_{\vec{p}_{1}, \sigma_{1}} \hat{a}_{\vec{p}+\vec{q}, \sigma} \mid \hat{a}_{p, \sigma}^{+}\right\rangle\right\rangle_{\omega_{n}}
\end{aligned}
$$

Здесь мы используем принятые обозначения для температурных функций Грина [33], в частности

$$
g_{a}\left(p, \sigma ; \omega_{n}\right) \equiv\left\langle\left\langle\hat{a}_{\vec{p}, \sigma} \mid \hat{a}_{\vec{p}, \sigma}^{+}\right\rangle\right\rangle_{\omega_{n}} .
$$

В силу условия квазинейтральности (4) в сумме по волновым векторам $\vec{q}$ в правой части равенства (23) исключен член с $\vec{q}=0$ [5]. Двухчастичная функция Грина в правой части равенства (23) после выделения так называемых "связных" частей [34], представляемых в виде произведения средних, может быть записана как

$$
\begin{aligned}
\left\langle\left\langle\hat{b}_{\vec{p}_{1}+\vec{q}, \sigma_{1}}^{+} \hat{b}_{\vec{p}_{1}, \sigma_{1}} \hat{a}_{\vec{p}+\vec{q}, \sigma} \mid \hat{a}_{\vec{p}, \sigma}^{+}\right\rangle\right\rangle_{\omega_{n}}= & \\
= & \left(f_{a}\left(p_{1}, \sigma_{1}\right) \delta_{\vec{q}, 0}-f_{a}(|\vec{p}+\vec{q}|, \sigma) \delta_{\vec{p}, \vec{p}_{1}} \delta_{\sigma, \sigma_{1}}\right) g_{a}\left(p, \sigma ; \omega_{n}\right) \delta_{a, b}+ \\
& +\left\langle\left\langle\hat{b}_{\vec{p}_{1}+\vec{q}, \sigma_{1}}^{+} \hat{b}_{p_{1}, \sigma_{1}} \hat{a}_{\vec{p}+\vec{q}, \sigma} \mid \hat{a}_{\vec{p}, \sigma}^{+}\right\rangle\right\rangle_{\omega_{n}}^{(\mathrm{reg})},
\end{aligned}
$$

где индекс (reg) означает, что данная функция является "регулярной" частью соответствующей функции Грина, а именно определена и совпадает с этой функцией Грина при $\vec{p}_{1} \neq \vec{p}$ и $\vec{q} \neq 0$, в ней отсутствуют "связные" части, и она не имеет особенностей при $\vec{p}_{1} \rightarrow \vec{p}$ и $\vec{q} \rightarrow 0$.

С учетом равенства (24) уравнение движения (23) для ОЧФГ $g_{a}\left(p, \sigma ; \omega_{n}\right)$ после перехода к термодинамическому пределу $V \rightarrow \infty$ принимает вид

$$
\left(i \omega_{n}-E_{a}(p, \sigma)+\mu_{a}\right) g_{a}\left(p, \sigma ; \omega_{n}\right)=1+\sum_{b} \int \frac{d^{3} q}{(2 \pi)^{3}} u_{a b}(q)\left\langle\left\langle\hat{\rho}_{\vec{q}}^{b} \hat{a}_{\vec{p}-\vec{q}, \sigma} \mid \hat{a}_{\vec{p}, \sigma}^{+}\right\rangle\right\rangle_{\omega_{n}}^{(\mathrm{reg})}
$$

где

$$
E_{a}(p, \sigma)=\epsilon_{a}(p)-\frac{z_{a}^{2} e^{2}}{\pi p} \int_{0}^{\infty} d q q f_{a}(q, \sigma) \ln \left|\frac{p+q}{p-q}\right|
$$

причем

$$
E_{a}(0, \sigma)=\lim _{p \rightarrow 0} E_{a}(p, \sigma)=-\frac{2 z_{a}^{2} e^{2}}{\pi} \int_{0}^{\infty} d q f_{a}(q, \sigma)<0 .
$$

Аналогично можно получить уравнение движения для "регулярной" части двухчастичной функции Грина, которое содержит ОЧФГ и соответствующие средние, а также "регулярные" части трехчастичных функций Грина и т. д. (см. статьи [34], [35]). Таким образом, в соответствии с предлагаемой процедурой низшее приближение для ОЧФГ соответствует тому, что мы пренебрегаем "регулярными" частями всех функций Грина более высокого порядка. Следующее приближение отвечает тому, что мы учитываем "регулярную" часть двухчастичной функции Грина и пренебрегаем "регулярной" частью трехчастичной функции Грина, и т. д. 
Для построения соответствующей процедуры при вычислении ДП используем соотношения (18), (19). При этом, как уже было отмечено ранее, задача сводится к определению поляризационного оператора “заряд-заряд” П $\left(q, i \omega_{l}\right)$ :

$$
\Pi\left(q, i \omega_{l}\right)=\sum_{a} z_{a} e \Pi_{a}\left(q, i \omega_{l}\right), \quad \Pi_{a}\left(q, i \omega_{l}\right) \equiv V^{-1}\left\langle\left\langle\hat{\rho}_{\vec{q}}^{a} \mid \hat{\rho}_{-\vec{q}}^{z}\right\rangle\right)_{\omega_{l}}^{(q)}
$$

где индекс $(q)$ обозначает, что соответствующая функция $\Pi_{a}\left(q, i \omega_{l}\right)$ является неприводимой частью функции отклика $\chi_{a z}\left(q, i \omega_{l}\right)$ по одной линии кулоновского взаимодействия. Согласно соотношению (27) справедливо равенство

$$
\Pi_{a}\left(q, i \omega_{l}\right)=V^{-1} \sum_{\vec{p}, \sigma} F_{a}\left(\vec{p}, \sigma ; \vec{q} ; i \omega_{l}\right),
$$

где

$$
F_{a}\left(\vec{p}, \sigma ; \vec{q} ; i \omega_{l}\right) \equiv\left\langle\left\langle\hat{a}_{\vec{p}-\vec{q} / 2, \sigma}^{+} \hat{a}_{\vec{p}+\vec{q} / 2, \sigma} \mid \hat{\rho}_{-q}^{z}\right\rangle\right\rangle_{\omega_{l}}^{(q)}
$$

Используя уравнения движения для операторов рождения и уничтожения, нетрудно убедиться, что функция $F_{a}\left(\vec{p}, \sigma ; \vec{q} ; i \omega_{l}\right)$ удовлетворяет уравнению (см. подробнее в работах [37], [38])

$$
\begin{aligned}
& \left(i \omega_{l}+\epsilon_{a}(|\vec{p}-\vec{q} / 2|)-\epsilon_{a}(|\vec{p}+\vec{q} / 2|)\right) F_{a}\left(\vec{p}, \sigma ; \vec{q} ; i \omega_{l}\right)= \\
& =z_{a} e\left(f_{a}(|\vec{p}-\vec{q} / 2|, \sigma)-f_{a}(|\vec{p}+\vec{q} / 2|, \sigma)\right)-V^{-1} \sum_{b} \sum_{\vec{k} \neq 0} u_{a b}(k) \Phi_{a b}\left(\vec{p}, \vec{k}, \sigma ; \vec{q} ; i \omega_{l}\right),
\end{aligned}
$$

где

$$
\Phi_{a b}\left(\vec{p}, \vec{k}, \sigma ; \vec{q} ; i \omega_{l}\right) \equiv\left\langle\left\langle\hat{a}_{\vec{p}-\vec{k}-\vec{q} / 2, \sigma}^{+} \hat{\rho}_{-\vec{k}}^{b} \hat{a}_{\vec{p}+\vec{q} / 2, \sigma}-\hat{a}_{\vec{p}-\vec{q} / 2, \sigma}^{+} \hat{\rho}_{\vec{k}}^{b} \hat{a}_{\vec{p}-\vec{k}+\vec{q} / 2, \sigma} \mid \hat{\rho}_{-\vec{q}}^{z}\right\rangle\right\rangle_{\omega_{l}}^{(q)}
$$

Наш дальнейший анализ связан с вычислением функции Грина $\Phi_{a b}\left(\vec{p}, \vec{k}, \sigma ; \vec{q} ; i \omega_{l}\right)$. Учтем, что согласно определению (28) функция Грина $F_{a}\left(\vec{p}, \sigma ; \vec{q} ; i \omega_{l}\right)$ является неприводимой по одной линии взаимодействия. Тем самым в $\Phi_{a b}\left(\vec{p}, \vec{k}, \sigma ; \vec{q} ; i \omega_{l}\right)$ отсутствуют члены, отвечающие условию $\vec{k}= \pm \vec{q}$. Далее по аналогии с проведенным выше рассмотрением ОЧФГ, выделяя в функции $\Phi_{a b}$ оставшиеся "связные" части, получаем

$$
\begin{aligned}
\Phi_{a b}\left(\vec{p}, \vec{k}, \sigma ; \vec{q} ; i \omega_{l}\right)= & -\left(f_{a}(|\vec{p}-\vec{k}-\vec{q} / 2|, \sigma)-f_{a}(|\vec{p}-\vec{k}+\vec{q} / 2|, \sigma)\right) F_{a}\left(\vec{p}, \sigma ; \vec{q} ; i \omega_{l}\right) \delta_{a, b}+ \\
& +\left(f_{a}(|\vec{p}-\vec{q} / 2|, \sigma)-f_{a}(|\vec{p}+\vec{q} / 2|, \sigma)\right) F_{a}\left(\vec{p}-\vec{k}, \sigma ; \vec{q} ; i \omega_{l}\right) \delta_{a, b}+ \\
& +\Phi_{a b}^{(\operatorname{reg})}\left(\vec{p}, \vec{k}, \sigma ; \vec{q} ; i \omega_{l}\right)
\end{aligned}
$$

Здесь индекс (reg) означает, что данная функция является "регулярной" частью соответствующей функции Грина, а именно определена и совпадает с этой функцией для волновых векторов $\vec{k} \neq \pm \vec{q}$, в ней отсутствуют "связные" части, и она не имеет особенностей в пределе $\vec{k} \rightarrow \pm \vec{q}$. 
С учетом соотношений (26), (30) после перехода к термодинамическому пределу $V \rightarrow \infty$ уравнение (29) принимает вид

$$
\begin{aligned}
& \left(i \omega_{l}+E_{a}(|\vec{p}-\vec{q} / 2|, \sigma)-E_{a}(|\vec{p}+\vec{q} / 2|, \sigma)\right) F_{a}\left(\vec{p}, \sigma ; \vec{q} ; i \omega_{l}\right)= \\
& =\left(f_{a}(|\vec{p}-\vec{q} / 2|, \sigma)-f_{a}(|\vec{p}+\vec{q} / 2|, \sigma)\right)\left(z_{a} e+\int \frac{d^{3} k}{(2 \pi)^{3}} u_{a a}(k) F_{a}\left(\vec{p}-\vec{k}, \sigma ; \vec{q} ; i \omega_{l}\right)\right)- \\
& \quad-\sum_{b} \int \frac{d^{3} k}{(2 \pi)^{3}} u_{a b}(k) \Phi_{a b}^{(\mathrm{reg})}\left(\vec{p}, \vec{k}, \sigma ; \vec{q} ; i \omega_{l}\right) .
\end{aligned}
$$

Продолжая эти рассуждения, мы можем получить цепочку уравнений для "регулярных" частей функций Грина, каждое из которых связывает "регулярную" часть функции Грина определенного порядка с "регулярными" частями функций Грина меньших порядков и соответствующими средними, а также с "регулярной" частью функции Грина следующего порядка. Таким образом, на основе выделения "связных" и "регулярных" частей можно построить процедуру получения бесконечной цепочки уравнений для различных температурных функций Грина, определяющих как ОЧФР, так и ДП.

\section{4. САМОСОГЛАСОВАННОЕ ПРИБЛИЖЕНИЕ ХАРТРИ-ФОКА}

Процедура разделения функций Грина на "связную" и "регулярную" части начиная с двухчастичной функции Грина, на наш взгляд, имеет непосредственное отношение к решению поставленной ранее задачи о выводе самосогласованных уравнений для ОЧФР и ДП. Действительно, если в соотношениях (25) и (31) пренебречь "регулярными" частями функций Грина более высокого порядка, мы получим замкнутые уравнения для функций $g_{a}\left(p, \sigma ; \omega_{n}\right)$ и $F_{a}\left(\vec{p}, \sigma ; \vec{q} ; i \omega_{l}\right)$, однозначно определяющие ОЧФР и ДП. В этом случае функция $g_{a}^{\text {SHFA }}\left(p, \sigma ; \omega_{n}\right)$ соответствует самосогласованному приближению Хартри-Фока (см. монографию [3]):

$$
g_{a}^{\mathrm{SHFA}}\left(p, \sigma ; \omega_{n}\right)=\frac{1}{i \omega_{n}-E_{a}^{\mathrm{SHFA}}(p, \sigma)+\mu_{a}},
$$

где

$$
\begin{aligned}
E_{a}^{\mathrm{SHFA}}(p, \sigma) & =\epsilon_{a}(p)-\frac{z_{a}^{2} e^{2}}{\pi p} \int_{0}^{\infty} d q q f_{a}^{\mathrm{SHFA}}(q, \sigma) \ln \left|\frac{p+q}{p-q}\right|, \\
f_{a}^{\mathrm{SHFA}}(p, \sigma) & =\frac{1}{\exp \left\{\left(E_{a}^{\mathrm{SHFA}}(p, \sigma)-\mu_{a}^{\mathrm{SHFA}}\right) / T\right\}+1} .
\end{aligned}
$$

Нетрудно убедиться, что эти соотношения образуют замкнутую систему уравнений для определения ОЧФР $f_{a}^{\text {SHFA }}(q, \sigma)$ или энергетического спектра одночастичных возбуждений $E_{a}^{\mathrm{SHFA}}(p, \sigma)$. Химический потенциал $\mu_{a}^{\mathrm{SHFA}}$ для частиц сорта $a$ в этом приближении определяется из условия нормировки для средней плотности числа частиц:

$$
n_{a}^{\mathrm{SHFA}}=\sum_{\sigma} \int \frac{d^{3} p}{(2 \pi)^{3}} f_{a}^{\mathrm{SHFA}}(p, \sigma) .
$$


Далее, согласно формуле (31) уравнение для функции $F_{a}^{\mathrm{SHFA}}\left(\vec{p}, \sigma ; \vec{q} ; i \omega_{l}\right)$ в рассматриваемом приближении записывается следующим образом: пусть

$$
F_{a}^{\mathrm{SHFA}}\left(\vec{p}, \sigma ; \vec{q} ; i \omega_{l}\right) \equiv z_{a} e \bar{F}_{a}^{\mathrm{SHFA}}\left(\vec{p}, \sigma ; \vec{q} ; i \omega_{l}\right),
$$

тогда для функции $\bar{F}_{a}^{\text {sнғA }}\left(\vec{p}, \sigma ; \vec{q} ; i \omega_{l}\right)$ мы имеем уравнение

$$
\begin{aligned}
\left(i \omega_{l}+\right. & \left.E_{a}^{\mathrm{SHFA}}(|\vec{p}-\vec{q} / 2|, \sigma)-E_{a}^{\mathrm{SHFA}}(|\vec{p}+\vec{q} / 2|, \sigma)\right) \bar{F}_{a}^{\mathrm{SHFA}}\left(\vec{p}, \sigma ; \vec{q} ; i \omega_{l}\right)= \\
= & \left(f_{a}^{\mathrm{SHFA}}(|\vec{p}-\vec{q} / 2|, \sigma)-f_{a}^{\mathrm{SHFA}}(|\vec{p}+\vec{q} / 2|, \sigma)\right) \times \\
& \times\left(1+\int \frac{d^{3} k}{(2 \pi)^{3}} u_{a a}(k) \bar{F}_{a}^{\mathrm{SHFA}}\left(\vec{p}-\vec{k}, \sigma ; \vec{q} ; i \omega_{l}\right)\right) .
\end{aligned}
$$

Тем самым, если ОЧФР $f_{a}^{\text {SHFA }}(q, \sigma)$ (или энергетический спектр одночастичных возбуждений $\left.E_{a}^{\mathrm{SHFA}}(p, \sigma)\right)$ известна, функция $F_{a}^{\mathrm{SHFA}}$ однозначно определяется интегральным уравнением (36).

Обратим внимание, что процедура аналитического продолжения с дискретного множества точек $\left\{i \omega_{l}\right\}$ на мнимой оси на верхнюю полуплоскость комплексных частот $z$, необходимая для определения ДП $\varepsilon(q, \omega)$ (см. соотношения $(18),(19))$, в данном случае сводится к замене $i \omega_{l}$ на $\hbar z, z \rightarrow \omega+i 0$. Таким образом, в соответствии с формулами (27), (35), (36) после перехода к термодинамическому пределу в рассматриваемом приближении мы имеем

$$
\varepsilon^{\mathrm{SHFA}}(q, \omega)=1-\sum_{a} u_{a a}(q) \Pi_{a a}^{\mathrm{SHFA}}(q, \omega),
$$

где

$$
\begin{aligned}
\Pi_{a a}^{\mathrm{SHFA}}(q, \omega) & =2 \int \frac{d^{3} p}{(2 \pi)^{3}} \Delta F_{a}(\vec{p}, \vec{q}, \omega) \Lambda_{a}(\vec{p}, \vec{q}, \omega), \\
\Delta F_{a}(\vec{p}, \vec{q}, \omega) & \equiv \frac{\Delta f_{a}(\vec{p}, \vec{q})}{\Delta E_{a}(\vec{p}, \vec{q})+\hbar(\omega+i 0)}, \\
\Delta f_{a}(\vec{p}, \vec{q}) & \equiv f_{a}^{\mathrm{SHFA}}(|\vec{p}-\vec{q} / 2|)-f_{a}^{\mathrm{SHFA}}(|\vec{p}+\vec{q} / 2|), \\
\Delta E_{a}(\vec{p}, \vec{q}) & \equiv E_{a}^{\mathrm{SHFA}}(|\vec{p}-\vec{q} / 2|)-E_{a}^{\mathrm{SHFA}}(|\vec{p}+\vec{q} / 2|) .
\end{aligned}
$$

Здесь и далее спиновые индексы опущены в силу рассмотрения нормальных систем, для которых ОЧФР и энергетический спектр одночастичных возбуждений не зависят от спиновых индексов. При этом функция $\Lambda_{a}(\vec{p}, \vec{q}, \omega)$ удовлетворяет уравнению

$$
\Lambda_{a}(\vec{p}, \vec{q}, \omega)=1-\int \frac{d^{3} k}{(2 \pi)^{3}} u_{a a}(|\vec{p}-\vec{k}|) \Delta F_{a}(\vec{k}, \vec{q}, \omega) \Lambda_{a}(\vec{k}, \vec{q}, \omega),
$$

которое известно в теории вырожденной электронной жидкости [39].

Далее нам необходимо убедиться, что представленные выше соотношения для ОЧФР и ДП в рамках самосогласованного приближения Хартри-Фока удовлетворяют одному из условий самосогласования (8). Согласно формулам (38)-(41)

$$
\lim _{q \rightarrow 0} \Pi_{a a}^{\mathrm{SHFA}}(q, 0)=2 \int \frac{d^{3} p}{(2 \pi)^{3}} \frac{\partial f_{a}^{\mathrm{SHFA}}(p)}{\partial E_{a}^{\mathrm{SHFA}}(p)} \bar{\Lambda}_{a}(p)
$$


где функция $\bar{\Lambda}_{a}(p)=\lim _{q \rightarrow 0} \Lambda_{a}(\vec{p}, \vec{q}, 0)$ удовлетворяет уравнению

$$
\bar{\Lambda}_{a}(p)=1-\int \frac{d^{3} k}{(2 \pi)^{3}} u_{a a}(|\vec{p}-\vec{k}|) \frac{\partial f_{a}^{\mathrm{SHFA}}(k)}{\partial E_{a}^{\mathrm{SHFA}}(k)} \bar{\Lambda}_{a}(k) .
$$

Учитывая далее формулы (33), находим

$$
\frac{\partial f_{a}^{\mathrm{SHFA}}(p)}{\partial E_{a}^{\mathrm{SHFA}}(p)} \bar{\Lambda}_{a}(p)=-\left(\frac{\partial f_{a}^{\mathrm{SHFA}}(p)}{\partial \mu_{a}^{\mathrm{SHFA}}}\right)_{T} .
$$

Подставляя это равенство в соотношения (34) и (43), убеждаемся, что в рамках в рамках самосогласованного приближения Хартри-Фока при произвольном вырождении выполняется предельное соотношение

$$
\lim _{q \rightarrow 0} \Pi_{a a}^{\mathrm{SHFA}}(q, 0)=-\left(\frac{\partial n_{a}^{\mathrm{SHFA}}}{\partial \mu_{a}^{\mathrm{SHFA}}}\right)_{T} .
$$

Таким образом, условие самосогласования (8) выполняется, поскольку при использовании самосогласованного приближения Хартри-Фока взаимодействие между частицами различных сортов не учитывается, но осуществляется самосогласованный учет взаимодействия между частицами одного сорта. Действительно, в этом случае одночастичная корреляционная функция равна [3] (см. равенство (32))

$$
g_{a}^{<}(p, \sigma ; \omega)=\frac{2 \pi}{\exp \left\{\left(\omega-\mu_{a}^{\mathrm{SHFA}}\right) / T\right\}+1} \delta\left(\omega-E_{a}^{\mathrm{SHFA}}(p)\right) .
$$

Подставляя это соотношение в (21), находим выражение для средней потенциальной энергии частиц сорта $а$ при произвольном вырождении в рамках самосогласованного приближения Хартри-Фока:

$$
\left\langle\widehat{U}_{a a}\right\rangle^{\mathrm{SHFA}}=-\int \frac{d^{3} p}{(2 \pi)^{3}} \int \frac{d^{3} k}{(2 \pi)^{3}} u_{a a}(|\vec{p}-\vec{k}|) f_{a}^{\mathrm{SHFA}}(p) f_{a}^{\mathrm{SHFA}}(k) .
$$

С другой стороны, для ДП справедливо высокочастотное разложение (см., например, работу [40])

$$
\left.\varepsilon(q, \omega)\right|_{\omega \rightarrow \infty}=1-\frac{\omega_{\mathrm{p}}^{2}}{\omega^{2}}-\frac{m_{2}(q)}{\omega^{4}}+o\left(\omega^{-4}\right),
$$

где

$$
\begin{aligned}
m_{2}(q)= & \sum_{a} \omega_{a}^{2}\left(\frac{2\left\langle T_{a}\right\rangle q^{2}}{m_{a}}+\frac{\hbar^{2} q^{4}}{4 m_{a}^{2}}\right)+\sum_{a, b}\left(n_{a} n_{b}\right)^{1 / 2} \int_{0}^{\infty} d k k^{2}\left(S_{a b}(k)-\delta_{a, b}\right) \times \\
& \times\left\{\frac{z_{a}^{2} z_{b}^{2} e^{4}}{m_{a} m_{b}}\left(\frac{\left(q^{2}-k^{2}\right)^{2}}{k q^{3}} \ln \left|\frac{q+k}{q-k}\right|-\frac{2 k^{2}}{q^{2}}+6\right)-\frac{8 z_{a}^{3} z_{a} e^{4}}{3 m_{a}^{2}}\right\} .
\end{aligned}
$$

Здесь $\omega_{\mathrm{p}}$ - плазменная частота, $\left\langle T_{a}\right\rangle$ - точная средняя кинетическая энергия, приходящаяся на одну частицу сорта $a$,

$$
\begin{gathered}
\omega_{\mathrm{p}}=\left(\sum_{a} \omega_{a}^{2}\right)^{1 / 2}, \quad \omega_{a}=\left(\frac{4 \pi z_{a}^{2} e^{2} n_{a}}{m_{a}}\right)^{1 / 2}, \\
\left\langle T_{a}\right\rangle=\frac{1}{n_{a}} \sum_{\sigma} \int \frac{d^{3} p}{(2 \pi)^{3}} \epsilon_{a}(p) f_{a}(p, \sigma) .
\end{gathered}
$$


При малых волновых векторах $q$ из формулы (46) следует, что

$$
m_{2}(q) \approx m_{2}(0)+\left(\sum_{a} \frac{2 \omega_{a}^{2}\left\langle T_{a}\right\rangle}{m_{a}}+\sum_{a, b} \frac{16 \pi z_{a} z_{b} e^{2}}{15 m_{a} m_{b}}\left\langle u_{a b}\right\rangle\right) q^{2}
$$

где

$$
\begin{gathered}
m_{2}(0)=\lim _{q \rightarrow 0} m_{2}(q)=\frac{8}{3} \sum_{a \neq b}\left(n_{a} n_{b}\right)^{1 / 2}\left(\frac{z_{a}^{2} z_{b}^{2} e^{4}}{m_{a} m_{b}}-\frac{z_{a}^{3} z_{a} e^{4}}{m_{a}^{2}}\right) \int_{0}^{\infty} d k k^{2} S_{a b}(k), \\
\left\langle u_{a b}\right\rangle \equiv \frac{\left\langle\widehat{U}_{a b}\right\rangle}{V}=\frac{1}{2}\left(n_{a} n_{b}\right)^{1 / 2} \int \frac{d^{3} k}{(2 \pi)^{3}} u_{a b}(k)\left(S_{a b}(k)-\delta_{a, b}\right) .
\end{gathered}
$$

Таким образом, используя высокочастотное разложение (45)-(50) для ДП, мы имеем возможность определить среднюю кинетическую и среднюю потенциальную энергии, соответствующие используемому приближению вычисления ДП. Для самосогласованного приближения Хартри-Фока данная задача была решена в работе [36] на основе высокочастотного разложения интегрального уравнения (42) с учетом соотношений (39)-(41). Полученное при этом выражение для средней потенциальной энергии совпадает с (44). Величина $\left\langle T_{a}\right\rangle$, заданная в (47), очевидно, равна

$$
\left\langle T_{a}\right\rangle^{\mathrm{SHFA}}=\frac{1}{n_{a}^{\mathrm{SHFA}}} \sum_{\sigma} \int \frac{d^{3} p}{(2 \pi)^{3}} \epsilon_{a}(p) f_{a}^{\mathrm{SHFA}}(p, \sigma) .
$$

Полученные выше результаты позволяют утверждать, что использование самосогласованного приближения Хартри-Фока в виде соотношений $(33),(34)$ для ОЧФР и соотношений (37)-(42) для ДП обеспечивает согласованное определение термодинамических свойств. Для установления пределов применимости этого приближения необходимо построить следующее самосогласованное приближение для ОЧФР, связанное с учетом "регулярной" части двухчастичной функции Грина в (24), а также самосогласованное приближение для ДП, связанное с учетом функции $\Phi_{a b}^{(\mathrm{reg})}$ в (31). Мы рассмотрим эти задачи в следующей публикации.

\section{5. ЗАКЛЮЧЕНИЕ}

В настоящей работе показано, что термодинамический потенциал Гиббса нерелятивистской системы заряженных частиц однозначно определяется ее диэлектрической проницаемостью и функциями распределения электронов и ядер вне рамок теории возмущений. Таким образом, при вычислении диэлектрической проницаемости и одночастичных функций распределения электронов и ядер следует применять согласованные приближения. Для решения этой задачи предлагается использовать процедуру расцепления, основанную на рассмотрении уравнений движения и выделении "связных" и "регулярных" частей температурных функций Грина. Показано, что простейшее приближение, отвечающее этой процедуре, соответствует самосогласованному приближению Хартри-Фока, в котором выполняются все известные условия самосогласования для диэлектрической проницаемости и функций распределения электронов и ядер. 
Благодарности. Автор выражает благодарность С. А. Тригеру за внимание и интерес к работе. Работа выполнена при поддержке РФФИ (грант № 12-08-00600_а).

\section{Список литературы}

[1] E. H. Lieb, R. Seiringer, The Stability of Matter in Quantum Mechanics, Cambridge Univ. Press, Cambridge, 2009.

[2] W.-D. Kraeft, D. Kremp, W. Ebeling, G. Ropke, Quantum Statistics of Charged Particle Systems, Plenum, New York, 1986.

[3] L.P. Kadanoff, G. Baym, Quantum Statistical Mechanics. Green's Function Methods in Equilibrium and Nonequilibrium Problems, Benjamin, New York, 1962.

[4] А. А. Абрикосов, Л. П. Горьков, И. Е. Дзялошинский, Методы квантовой теории поля в статистической физике, Физматгиз, М., 1962.

[5] V. B. Bobrov, I. M. Sokolov, S. A. Trigger, Phys. Plasmas, 19:6 (2012), 062101, 8 pp.

[6] T. Matsubara, Progr. Theoret. Phys., 14:4 (1955), 351-378.

[7] А. А. Веденов, А. И. Ларкин, ЖЭТФ, 36:4 (1959), 1133-1142.

[8] А.Н. Старостин, В. К. Рерих, ЖЭТФ, 127:1 (2005), 186-219.

[9] D. Kremp, M. Schlanges, W.-D. Kraeft, Quantum Statistics of Nonideal Plasmas, Atomic, Optical, and Plasma Physics, 25, Springer, Berlin, 2005.

[10] D. Pines, P. Nozières, The Theory of Quantum Liquids, Benjamin, New York, 1966.

[11] R. Redmer, G. Röpke, Contr. Plasma Phys., 50:10 (2010), 970-985.

[12] E. Montroll, J. Ward, Phys. Fluids, 1 (1958), 55-72.

[13] Ю. Г. Красников, ЖЭТФ, 53:6 (1967), 2223-2232.

[14] F. J. Rogers, H. E. DeWitt, Phys. Rev. A, 8:2 (1973), 1061-1076.

[15] A. Alastuey, V. Ballenegger, F. Cornu, Ph. A. Martin, J. Stat. Phys., 130:6 (2008), 1119-1176.

[16] A. Alastuey, V. Ballenegger, Phys. Rev. E, 86:6 (2012), 066402, 20 pp.

[17] E. W. Brown, B. K. Clark, J. L. DuBois, D. M. Ceperley, Phys. Rev. Lett., 110:14 (2013), 5, 146405.

[18] Y. A. Omarbakiyeva, C. Fortmann, T. S. Ramazanov, G. Röpke, Phys. Rev. E, 82:2 (2010), 026407, 14 pp., arXiv: 1003.4459.

[19] W. Ebeling, W. D. Kraeft, G. Röpke, Contr. Plasma Phys., 52:1 (2012), 7-16.

[20] W. Ebeling, W. D. Kraeft, G. Röpke, Ann. Phys. (Berlin), 524:6007 (2012), 311-326.

[21] В. Б. Бобров, Н. И. Ключников, С. А. Тригер, ТМФ, 89:2 (1991), 263-277.

[22] V. B. Bobrov, N. I. Klyuchnikov, S. A. Trigger, Physica A, 181:1-2 (1992), 150-172.

[23] Д. Н. Зубарев, Неравновесная статистическая термодинамика, Наука, М., 1971.

[24] V.B. Bobrov, S. A. Trigger, G. J. F. van Heijst, P. P. J. M. Schram, Phys. Rev. E, 82:1 (2010), 010102(R), 3 pp.

[25] V. B. Bobrov, S. A. Trigger, A. Zagorodny, Phys. Rev. A, 82:4 (2010), 044105, 4 pp.

[26] V. B. Bobrov, J. Phys.: Cond. Matt., 2:13 (1990), 3115-3118.

[27] V.B. Bobrov, S. A. Trigger, Virial theorem and Gibbs thermodynamic potential for Coulomb systems, arXiv: 1310.7703.

[28] J. M. Luttinger, J. C. Ward, Phys. Rev., 118:5 (1960), 1417-1427.

[29] M. Potthoff, M. Aichhorn, C. Dahnken, Phys. Rev. Lett., 91:20 (2003), 206402, 4 pp.

[30] M. Potthoff, M. Balzer, Phys. Rev. B, 75:12 (2007), 125112, 22 pp., arXiv: cond-mat/0610217.

[31] H. Suhl, N. Werthamer, Phys. Rev., 122:2 (1960), 359-366.

[32] E. R. Caianiello (ed.), Lectures on the Many-Body Problem, v. 2, Academic Press, New York, 1964. 
[33] Д. Н. Зубарев, УФН, 71:5 (1960), 71-116.

[34] В. Д. Озрин, ТМФ, 4:1 (1970), 66-75.

[35] В. Б. Бобров, Теплофизика высоких температур, 32:4 (1994), 497-502.

[36] В. Б. Бобров, Теплобизика высоких температур, 33:6 (1995), 867-573.

[37] В. Б. Бобров, Р. Редмер, Г. Репке, С. А. Тригер, ТМФ, 86:2 (1991), 300-311.

[38] В. Б. Бобров, Р. Редмер, Г. Репке, С. А. Тригер, ТМФ, 86:3 (1991), 425-437.

[39] В. Д. Горобченко, Е. Г. Максимов, УФН, 130:1 (1980), 65-111.

[40] В. Б. Бобров, ЖЭТФ, 102:6 (1992), 1808-1815.

Поступила в редакцию 14.01.2014 